\title{
Cooperative Sensing With WIBA Energy Detection Under Rural Area Channel Conditions
}

\author{
Johanna Vartiainen \\ Centre for Wireless Communications \\ University of Oulu \\ Oulu, Finland \\ johanna.vartiainen@oulu.fi
}

\author{
Alexandre Matos \\ Federal University of Ceara \\ Brazil \\ alexandrematos@gtel.ufc.br
}

\author{
Heikki Karvonen \\ Centre for Wireless Communications \\ University of Oulu \\ Oulu, Finland \\ heikki.karvonen@oulu.fi
}

\author{
Marja Matinmikko-Blue \\ Centre for Wireless Communications \\ University of Oulu \\ Oulu, Finland \\ marja.matinmikko@oulu.fi
}

\begin{abstract}
Remote and rural area connectivity is a true challenge that can be alleviated by allowing shared spectrum access in bands below $1 \mathrm{GHz}$. Spectrum sensing can provide benefits when used together with the database approach for realizing spectrum sharing. Energy detection (ED) is very suitable in cooperative sensing because of its low computational complexity and it does not need prior knowledge about the signal and noise. In this work, cooperative sensing using the window-based (WIBA) ED method is studied to maximize signal detection distance in a rural area scenario with a dedicated channel model. Based on the required individual user detection probabilities, cooperative signal detection distances in kilometers are explored using both OR and $k$-out-of- $n$-rules. The results are compared to that of the localization algorithm based on double-thresholding (LAD) method. Computer simulations using a rural area channel model show that the detection distance difference is tens of kilometers. Furthermore, it was found that the signal detection distance improvement can be even five-fold when using the cooperative sensing approach. Thus the proper use and design of cooperative sensing can help in rural area connectivity.
\end{abstract}

Keywords-spectrum sensing, collaborative sensing, signal detection, remote area, channel model.

\section{INTRODUCTION}

Connectivity in rural and remote areas continues to be a global challenge. These areas require the use of lower carrier frequencies below $1 \mathrm{GHz}$ to obtain sufficient network coverage. In places where mobile network operators (MNOs) lack the incentives to deploy cellular networks, license-exempt shared use of TV bands becomes an option to enable costefficient solution for rural area users which could operate as a secondary user utilizing spectrum white spaces. Spectrum sharing is therefore an enabler for the deployment of mobile communication networks to provide rural and remote area connectivity in a cost-feasible manner. Incumbent spectrum usage in these areas is often lower than in dense urban areas which provides opportunities for shared spectrum access while protecting the incumbents. Currently the rules developed for the use of TV white spaces (TVWS) typically rely on the use of geolocation database as the means to protect the incumbent TV broadcasting usage requiring that devices wishing to access the

\author{
Carlos Silva \\ Federal University of Ceara \\ Brazil \\ cfms@gtel.ufc.br
}

TVWSs need to get the permission to transmit from a database. That ensures the protection of the incumbents from harmful interference. The use of spectrum sensing techniques can provide benefits when used in conjunction with the database approach to allow shared spectrum access. Especially in rural and remote areas, spectrum sensing can be used to complement the database approach by bringing more accurate information on the actual spectrum usage in the given area. The use of a single node for conducting spectrum sensing is challenged by the hidden node problem which is why in rural and remote deployments the use of cooperative (collaborative) spectrum sensing is preferred [1], [2]. Long distances and varying terrains are the challenge in rural areas and therefore determining the sensing ranges is of key importance.

There exists several spectrum sensing techniques like energy detection (ED), matched filter detection, compressive sensing and cyclostationary detection [3]. ED [4] is widely studied and also adopted in practice due to its low computational complexity and low amount of prior knowledge need about the signal to be detected and noise. Even though ED sensing sensitivity performance is not among the best ones, cooperative sensing helps to overcome performance degradation [5].

In this paper, the performance of the window-based (WIBA) ED [6] used for cooperative sensing to maximize signal detection distance (or radius of the detection area) is investigated in a rural area case with a dedicated measurementbased channel model. Recently proposed WIBA ED with the adaptive sensing threshold has been shown to be among the most efficient ED methods [6]. It is able to estimate the noise level blindly and it operates even with signal-to-noise ratio (SNR) below $-10 \mathrm{~dB}$. Cooperative detection decision rules are introduced to define required individual user detection probabilities to get the final cooperative detection probability $P_{d} \geq 0.9(90 \%)$ [3]. Usually, only detection probabilities are studied in the literature. Here, also a detection distance is explored. Detection distance is defined here to be the maximum distance between the transmitter (TX) and the receiver (RX) to still achieve the final cooperative detection probability 
$P_{d} \geq 0.9$. The detection distance is a very important aspect especially in rural and remote areas, where distances are long. The effect of the used cooperative decision rule and the number of cooperative nodes to the detection distance are studied. Based on required individual user detection probabilities, signal detection distances in kilometers between the TX and RX are defined. Matlab simulations are performed using a rural area channel model. This is the first time when the WIBA method performance is evaluated for cooperative sensing case, and using a dedicated channel model for rural area scenario. The results can be used to enable proper design of cooperative spectrum sensing which helps to improve connectivity in remote areas together with the database approach. The results are compared to that of the well-known localization algorithm based on double-thresholding (LAD) ED [7], which outperforms conventional ED methods [8].

This paper is organized as follows. Section II describes the used rural area channel model. Section III describes cooperative detection and decision rules. Section IV introduces the used WIBA ED method. In Section VI, cooperative sensing results when using rural area channel model are presented, and Section VII presents our conclusions.

\section{Rural Area Channel Model}

The used channel model is based on measurements of delay spread and path loss performed in rural area by the companies Ericsson and Telstra for the carrier frequency of $850 \mathrm{MHz}$ and distance up to $200 \mathrm{~km}$ [9]. Restricting the measured data to the coverage area of $50 \mathrm{~km}$ and considering the carrier frequency of $700 \mathrm{MHz}$, a simpler path loss model was proposed

$$
\operatorname{PL}(d, f)=\operatorname{FSPL}(d, f)+K,
$$

where $d$ is the distance, $f$ is the central frequency, $\operatorname{FSPL}(\cdot)$ is the Free Space path loss model, and $K$ is an offset which minimizes the mean squared error (MSE) between the proposed path loss in (1) and the measured path loss samples in [9]. Fig. 1 depicts the measured data and the proposed path loss model where $K=29.38 \mathrm{~dB}$.

After the PL is determined, the shadow fading (SF), $\sigma_{\mathrm{SF}}$, can also be derived. Knowing that the SF corresponds to the large scale fluctuations of the Channel Impulse Response (CIR) around its average or, in other words, the standard deviation of the error function between the measured data and the proposed model (1), as in [10] it equals $\sigma_{\mathrm{SF}}=4.47 \mathrm{~dB}$. Moreover, the recommendations from [11] and the measured delay spread in [9] were used with the 3rd Generation Partnership Project (3GPP) Clustered Delay Line (CDL)-A model to generate the Small Scale Parameters (SSPs) [12] used in the channel model.

\section{CoOperative SEnsing}

In cooperative sensing, information from severe spatially distributed radios (or their antenna components) is used. The major reasons to use cooperative spectrum sensing are fading, Doppler effect, shadowing and receiver uncertainty problems [2], [3], [13]. This helps to prevent so called hidden terminal problem, where the radio is not able to sense the signal because of low SNR caused by shadowing. The problems are illustrated in Fig. 2, where user A is outside the primary user's (PU) transmission range and can not detect PU. In addition, there

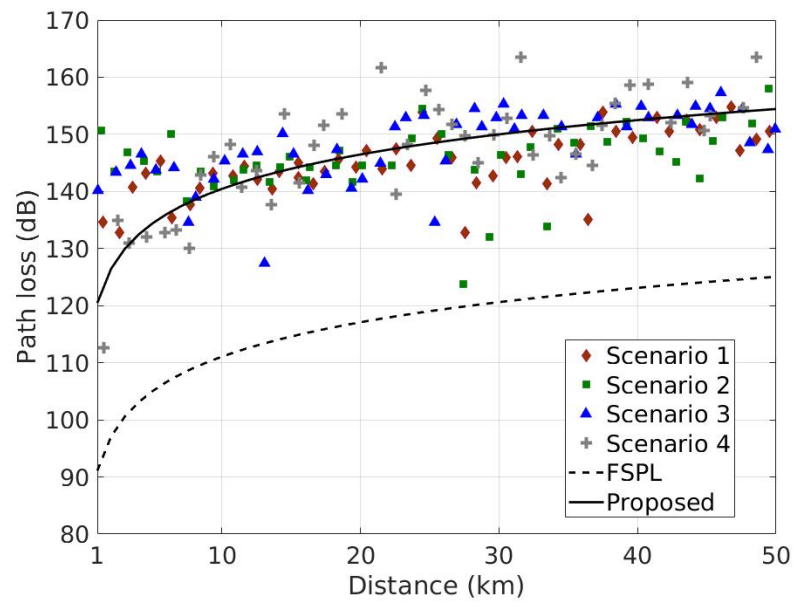

Figure 1. Measured data for four scenarios from [9] and the proposed path loss model. The measured data is composed of 191 points, where each point was obtained by averaging thousands of samples.

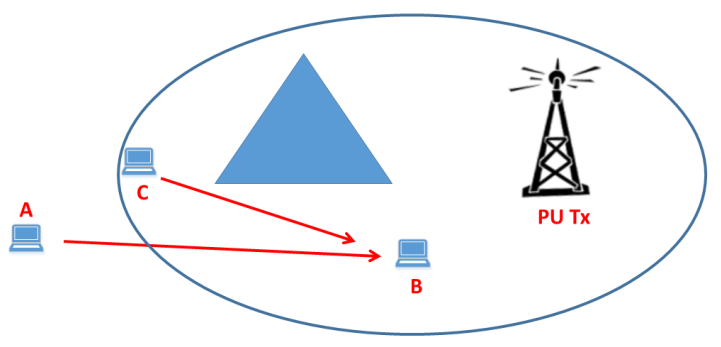

Figure 2. Illustration of hidden terminal problem in case of primary user (PU) and users A, B and C.

is a natural obstacle (mountain) between user $\mathrm{C}$ and PU, so $\mathrm{C}$ is not able to detect PU even though $\mathrm{C}$ is inside PUs transmission area. Another benefit of cooperative sensing is that single user's probability of detection performance can be lower when compared to the non-cooperative system. Because cooperation gives diversity gain, simple energy detection is the most common detection technique in cooperative sensing, even though its performance in non-cooperative detection is worse when compared to other techniques [3].

Cooperative sensing can be done in a distributed or centralized fashion [14]. In distributed sensing, radios operate independently and communicate among themselves. Local sensing results are shared with other radios, and decisions are made locally. In centralized cooperation, the sensing process is coordinated by a fusion center (FC) and the final sensing decision is transmitted to all cooperative radios.

In cooperative sensing there are two indicators, namely the detection probability $P_{d}$ and the false-alarm probability $P_{f}$. In spectrum sensing it is desired that the detection probability $P_{d}$ is as large as possible. Here, we are using a typical requirement that $P_{d} \geq 0.9$ [15]. It is also desired that false alarm probability $P_{f}$ is as small as possible. In the case of cooperative sensing, 
$P_{d}\left(P_{f}\right)$ means here the final cooperative detection (false alarm rate) probability.

Let us assume that individual detection probability for one node is $P_{d, i}$ and there are $n$ cooperating nodes which perform sensing and provide results to FC (at base station). Let us also assume that all the nodes have same individual $P_{d, i}$, i.e., $P_{d, 1}=P_{d, 2}=\cdots=P_{d, n}$. We assume a hard decision (HD) in which each node decides if the signal is present or not, and sends a one-bit information about the decision ( 0 or 1$)$ to the FC. HD selection is a compromise which decreases amount of data that mobile users need to send to FC, but on the other hand it decreases the accuracy of joint decision made by FC. Final cooperative detection probability $P_{d}$ depends on the number of cooperating nodes $n$ and also on the used decision rule. The general decision rules at FC are $k$-out-of- $n$, majority, OR and AND rules [1], [16], [17].

$k$-out-of- $n$ rule means that the signal is decided to be present in the channel if $k$ users out of $n$ users report that there is a signal at the detector input. In this case, the final detection probability at the FC is $P_{d}=\sum_{i=k}^{n}\left(\begin{array}{c}n \\ i\end{array}\right) P_{d, i}^{k}\left(1-P_{d, i}\right)^{n-i}[1]$, [18]. Corresponding final cooperative false alarm probability is $P_{f}=\sum_{i=k}^{n}\left(\begin{array}{c}n \\ i\end{array}\right) P_{f, i}^{k}\left(1-P_{f, i}\right)^{n-i}$ [1]. When $k=1$, this corresponds to OR rule (1-out-of- $n$ ), when $k=n$, this corresponds AND rule ( $n$-out-of- $n$ ), and when $k=n / 2$, this corresponds so called majority rule ( $[n / 2]$-out-of- $n$, called later as $[n / 2] / n$ rule) [3]. OR rule means that the signal is decided to be present when any of the cooperative sensing nodes reports that signal is detected. This means that final cooperative detection and false alarm rates are both high. In AND rule, the signal is decided not to be present even though only one cooperative node reports that the signal is not present. This means that final cooperative detection and false alarm rates are both small, so when using AND rule, cooperative detection does not increase the detection probability. Optimal decision rule depends on, e.g., the number of $n$, channel and used detection threshold [3]. Majority rule has been noticed to be optimal in most of the cases [19], so it is used also here when exploring the detection distance.

It is reasonable to assume that in rural area scenario, the amount of possible cooperative nodes is small. Therefore, in this paper $n$ was selected to be 5,7 and 10 . Table I presents which individual user detection probability values will result in the final cooperative detection probability to be $P_{d} \geq 0.9$ when using $k$-out-of- $n$ rule. It can be noticed that larger the $n$ is, the smaller individual detection probability is required, and the better is the sensing performance. However, also $P_{f}$ increases with $n$ [1]. In addition, as $n$ increases, the reporting time and the overhead is higher, which also increases the energy consumption. So, it is not always reasonable - or even possible, e.g., in rural areas - to have as many cooperative nodes as possible. From Table I it can also be seen that large $k$ requires high individual detection probability $P_{d, i}$ to get final cooperative $P_{d} \geq 0.9$. Majority rule has been found to be a good compromise between required individual detection probability and resulting cooperative detection performance [19]. When using majority rule, required individual user detection probability values $P_{d, i}$ are 0.8 for $3 / 5$ rule, 0.7 for $4 / 7$ rule, and 0.65 for $5 / 10$ rule (Table I). Those individual user detection probability values were used in the simulations. Also OR rule was used in the simulations so that the maximum
TABLE I. INDIVIDUAL $P_{d, i}:$ S TO GET FINAL COOPERATIVE $P_{d} \geq 0.9$ WHEN USING $k$-OUT-OF- $n$ RULE $(n=5,7$ AND 10$)$

\begin{tabular}{ll|ll|ll}
$k / 5$ & $P_{d, i}$ & $k / 7$ & $P_{d, i}$ & $k / 10$ & $P_{d, i}$ \\
\hline $1 / 5=\mathrm{OR}$ & 0.4 & $1 / 7=\mathrm{OR}$ & 0.3 & $1 / 10=\mathrm{OR}$ & 0.2 \\
$2 / 5$ & 0.6 & $2 / 7$ & 0.45 & $2 / 10$ & 0.35 \\
$3 / 5=$ MAJ. $^{*}$ & 0.8 & $3 / 7$ & 0.6 & $3 / 10$ & 0.45 \\
$4 / 5$ & 0.9 & $4 / 7=$ MAJ. $^{*}$ & 0.7 & $4 / 10$ & 0.55 \\
$5 / 5=$ AND & 0.98 & $5 / 7$ & 0.85 & $5 / 10=$ MAJ. $^{*}$ & 0.65 \\
& & $6 / 7$ & 0.95 & $6 / 10$ & 0.75 \\
& & $7 / 7=\mathrm{AND}$ & 0.98 & $7 / 10$ & 0.8 \\
& & & & $8 / 10$ & 0.9 \\
& & & & $9 / 10$ & 0.95 \\
* majority rule $^{*}$ m & & & & &
\end{tabular}

distances were obtained for comparison.

\section{THE WIBA METHOD}

In this work, spectrum sensing is performed using the WIBA method [6] that uses a detection threshold when defining if there is a signal or only noise present in the channel. If the signal is present, it means that the channel is occupied, as a noise-only case means that the channel is free for a secondary user transmission. Let us assume that the received frequency domain samples $x_{i}$ are zero mean, independent Gaussian distributed complex random variables. Thus, the sample energy $y_{i}=\left|x_{i}\right|^{2}$ follows a chi-squared distribution with $2 M$ degrees of freedom. The detection threshold parameter $T$ can be solved from [20], [21]

$$
P_{\mathrm{FA}}=e^{-T M} \sum_{k=0}^{M-1} \frac{1}{k !}(T M)^{k},
$$

where $P_{\mathrm{FA}}$ means the desired false alarm rate which is decided beforehand. When $M=1$, variables follow chi-squared distribution with two degrees of freedom, and from (2) we get that

$$
T_{\mathrm{CME}}=-\ln \left(P_{\mathrm{FA}}\right) \text {. }
$$

For example, if $M=1$ and $P_{\mathrm{FA}}=0.01, T=4.6$. The threshold parameter is constant for specific $M$ and $P_{\mathrm{FA}}$, and, thus, can be calculated beforehand.

In the WIBA method, at first the received waveform samples are divided into $L$ blocks called as detection windows with length $M$. Window length $M$ is selected beforehand. Here, $50 \%$ overlapping is used. It means that first block consist samples $1, \cdots, M$, second block consists samples $\frac{M}{2}+$ $1, \cdots, \frac{M}{2}+M$, third block consists samples $M+1, \cdots, 2 M$, and so on. Energy samples $y_{i}$ in each block are summed up to get the total energy in each block, which is, $Z_{i}=\sum_{l=1}^{M} Y_{i}(l)$, $i=1, \cdots, L$. The signal detection threshold is [6]

$$
T_{h}=T \frac{1}{L} \sum_{i=1}^{L} Z_{i},
$$

where $T$ comes from (2).

The LAD method [7] [8] is used as a point of comparison in the performance evaluation and therefore it is shortly introduced here. The LAD method uses iterative forward consecutive mean excision (FCME) threshold setting process [22]. The threshold is $T_{h}=T_{\mathrm{CME}} \bar{y}$, where $T_{\mathrm{CME}}$ comes from (3) and $\bar{y}$ is the mean. Basically, the threshold calculation corresponds to 
TABLE II. THE MAXIMUM DISTANCE BETWEEN TX AND RX WHERE THE SIGNAL CAN BE DETECTED FOR DIFFERENT TRANSMIT POWER VALUES [DBM] AND SIGNAL BANDWIDTHS [MHZ]. OR RULE IS USED. NUMBER OF COOPERATING NODES $n \geq 5$ AND $P_{d, i} \geq 0.4$.

\begin{tabular}{c|c|l|l}
$\begin{array}{c}\text { Transmit power } \\
{[\mathrm{dBm}]}\end{array}$ & $\begin{array}{c}\text { Signal BW } \\
{[\mathrm{MHz}]}\end{array}$ & $\begin{array}{l}\text { TX-RX distance } \\
\text { WIBA method }\end{array}$ & $\begin{array}{l}\text { TX-RX distance } \\
\text { LAD method }\end{array}$ \\
\hline 53 & 2 & $60 \mathrm{~km}$ & $<32 \mathrm{~km}$ \\
53 & 4 & $60 \mathrm{~km}$ & $<14 \mathrm{~km}$ \\
53 & 6 & $60 \mathrm{~km}$ & $<7 \mathrm{~km}$ \\
\hline 46 & 2 & $60 \mathrm{~km}$ & $<16 \mathrm{~km}$ \\
46 & 4 & $<45 \mathrm{~km}$ & $<6 \mathrm{~km}$ \\
46 & 6 & $<30 \mathrm{~km}$ & $<3 \mathrm{~km}$ \\
\hline 30 & 2 & $<12 \mathrm{~km}$ & $<2 \mathrm{~km}$ \\
30 & 4 & $<8 \mathrm{~km}$ & $<1 \mathrm{~km}$ \\
30 & 6 & $<5 \mathrm{~km}$ & - \\
\hline 20 & 2 & $<4 \mathrm{~km}$ & - \\
20 & 4 & $<3 \mathrm{~km}$ & - \\
20 & 6 & $<2 \mathrm{~km}$ & - \\
\hline 10 & 2 & $<1 \mathrm{~km}$ & - \\
10 & 4 & - & -
\end{tabular}

WIBA threshold setting when one sample corresponds to one block, i.e., detection window length $M=1$ and the number of blocks $L$ equals to the number of considered samples. The LAD method calculates two FCME thresholds, namely the upper and lower ones, with two different threshold parameters. After that, the LAD method clusters together adjacent samples above the lower threshold. The cluster is decided to be caused by a signal if at least one of the samples is also above the upper threshold. Usually, ACC parameter that allows $p$ (usually $p=3$ ) samples to be below the lower threshold between two accepted clusters, is used [8]. That procedure increases the detection performance.

\section{Simulations}

This section provides cooperative sensing results when using rural area channel model described in Section II. The total bandwidth of the channel is $23.4 \mathrm{MHz}$ and carrier frequency is $700 \mathrm{MHz}$. In the simulations, used transmit power values were $10,20,30,46$ or $53 \mathrm{dBm}$ and signal $\mathrm{BW}$ was 2,4 or $6 \mathrm{MHz}$ corresponding to $8.5,17$ and $25.6 \%$ of the total BW. Matlab simulations for the WIBA and LAD methods were performed to get individual detection probabilities $P_{d, i}$, and those results were combined to results from Section III in order to get final cooperative detection probability $P_{d}$ results (Table I). The results were used to find out sensing distances in kilometers $(\mathrm{km})$. Technically, sensing distance corresponds a radius of a circular detection area (or zone). For the sake of simplicity, this radius is called as a detection distance here. It is of interest what is the maximum distance [km] between TX and RX where signal still can be detected, assuming different signal BWs and transmit powers. It is assumed here that the signal is defined to be detected only if final cooperative detection probability $P_{d} \geq 0.9$ is achieved.

We used two cooperative decision rules, OR and majority $([n / 2] / n)$ rule. As the OR rule gives the best possible performance (so called upper limit of detection) but has high false alarm rate, majority rule is a good compromise and, thus, a reasonable choice that gives practical results. AND rule was not used because it requires that all the nodes must detect the signal, leading to a very low cooperative detection probability.

First, the results for OR rule are presented. In this case, the final cooperative $P_{d} \geq 0.9$ is reached when $n \geq 5$ and
TABLE III. THE MAXIMUM DISTANCE BETWEEN TX AND RX WHERE THE SIGNAL CAN BE DETECTED FOR DIFFERENT TRANSMIT POWER VALUES [DBM] AND SIGNAL BANDWIDTHS [MHz]. $k-o u t-o f-n$ RULE IS USED AND $P_{d, i} \geq 0.9$.

\begin{tabular}{c|c|c|l|l|l|l|l} 
& & \multicolumn{3}{|c|}{ TX-RX distance $[k m]$} & \multicolumn{3}{|c}{ TX-RX distance $[k m]$} \\
$\begin{array}{c}\text { Transmit } \\
\text { power } \\
{[\mathrm{dBm}]}\end{array}$ & $\begin{array}{c}\text { Signal } \\
\text { BW } \\
{[\mathrm{MHz}]}\end{array}$ & $\begin{array}{c}3 / 5 \\
\text { rule }\end{array}$ & $\begin{array}{l}4 / 7 \\
\text { rule }\end{array}$ & $\begin{array}{l}5 / 10 \\
\text { rule }\end{array}$ & $\begin{array}{l}3 / 5 \\
\text { rule }\end{array}$ & $\begin{array}{l}4 / 7 \\
\text { rule }\end{array}$ & $\begin{array}{l}5 / 10 \\
\text { rule }\end{array}$ \\
\hline 53 & 2 & 50 & $>60$ & $>60$ & 10 & 15 & 17 \\
53 & 4 & 33 & 44 & 50 & 4 & 6 & 7 \\
53 & 6 & 22 & 31 & 35 & 2 & 3 & 3.5 \\
\hline 46 & 2 & 24 & 32 & 40 & 4 & 7 & 8 \\
46 & 4 & 15 & 21 & 23 & 2 & 2.5 & 3 \\
46 & 6 & 10 & 14 & 17 & - & 1.5 & 1.5 \\
\hline 30 & 2 & 4 & 5 & 6 & - & 1 & 1 \\
30 & 4 & 2 & 3 & 4 & - & - & - \\
30 & 6 & 1 & 2 & 2.5 & - & - & - \\
\hline 20 & 2 & 1 & 1.5 & 2 & - & - & - \\
20 & 4 & - & 1 & 1 & - & - & - \\
20 & 6 & - & - & - & - & - & -
\end{tabular}

TABLE IV. PERFORMANCE IMPROVEMENT [\%] USING COOPERATIVE OR AND $k-o u t-o f-n$ RULES. THE WIBA METHOD.

\begin{tabular}{l|l|r|r|r|r}
$\begin{array}{l}\text { Transmit } \\
\text { power } \\
{[d B m]}\end{array}$ & $\begin{array}{l}\text { Signal } \\
\text { BW } \\
{[M H z]}\end{array}$ & $\begin{array}{r}\text { OR } \\
\text { rule }\end{array}$ & $\begin{array}{r}3 / 5 \\
\text { rule }\end{array}$ & $\begin{array}{r}4 / 7 \\
\text { rule }\end{array}$ & $\begin{array}{r}5 / 10 \\
\text { rule }\end{array}$ \\
\hline 53 & 2 & $170 \%$ & $147 \%$ & $176 \%$ & $176 \%$ \\
53 & 4 & $300 \%$ & $165 \%$ & $220 \%$ & $250 \%$ \\
53 & 6 & $400 \%$ & $200 \%$ & $200 \%$ & $230 \%$ \\
\hline 46 & 2 & $400 \%$ & $160 \%$ & $213 \%$ & $267 \%$ \\
46 & 4 & $500 \%$ & $170 \%$ & $233 \%$ & $255 \%$ \\
46 & 6 & $500 \%$ & $170 \%$ & $233 \%$ & $283 \%$
\end{tabular}

$P_{d, i} \geq 0.4$ (Table I). Table II presents the maximum distance [km] between TX and RX where the signal is detected. The WIBA method defeats the LAD method. It can be seen that when the WIBA method is used and transmit power is 53 $\mathrm{dBm}$, the distance where the signal can be detected is $60 \mathrm{~km}$, regardless of the signal BW. The corresponding distance for the LAD method is only $7-32 \mathrm{~km}$.

Next, the results for $3 / 5,4 / 7$ and $5 / 10$ rules are presented. In this case, the final cooperative $P_{d} \geq 0.9$ is reached when $P_{d, i}$ is 0.8 ( $3 / 5$ rule), 0.7 ( $4 / 7$ rule), and 0.65 (5/10 rule) (Table I). Table III presents the maximum distance $[\mathrm{km}]$ between TX and RX where the signal is detected. Again, the WIBA method outperforms the LAD method. For example, when transmit power is $53 \mathrm{dBm}$, signal $\mathrm{BW}$ is $2 \mathrm{MHz}$ and the WIBA method is used, signal can be detected (final cooperation detection probability $P_{d}=0.9$ ) when TX-RX distance is $50-60 \mathrm{~km}$. The corresponding distance for the LAD method is only $10-17 \mathrm{~km}$. It can be seen that when $n$ increases, also the detection distances increase. When the number of cooperative nodes is doubled, the detection distance is $10-17 \mathrm{~km}$ longer. However, it can be seen that even with few cooperative nodes $n$, it is possible to achieve long detection distances, even $50 \mathrm{~km}$, depending on the transmit power and signal bandwidth.

Table IV presents performance improvement in percentage terms. For example, when signal $\mathrm{BW}$ is $4 \mathrm{MHz}$ and transmit power is $53 \mathrm{dBm}$, cooperative sensing gives $300 \%$ (OR rule), $165 \%$ (3/5 -rule), $220 \%$ (4/7 -rule) and $250 \%$ (5/10 -rule) benefit. The larger the $n$ is, the bigger the performance difference is in per cent. The performance difference is between $147-500 \%$. That is, the detection distance is at least one and 


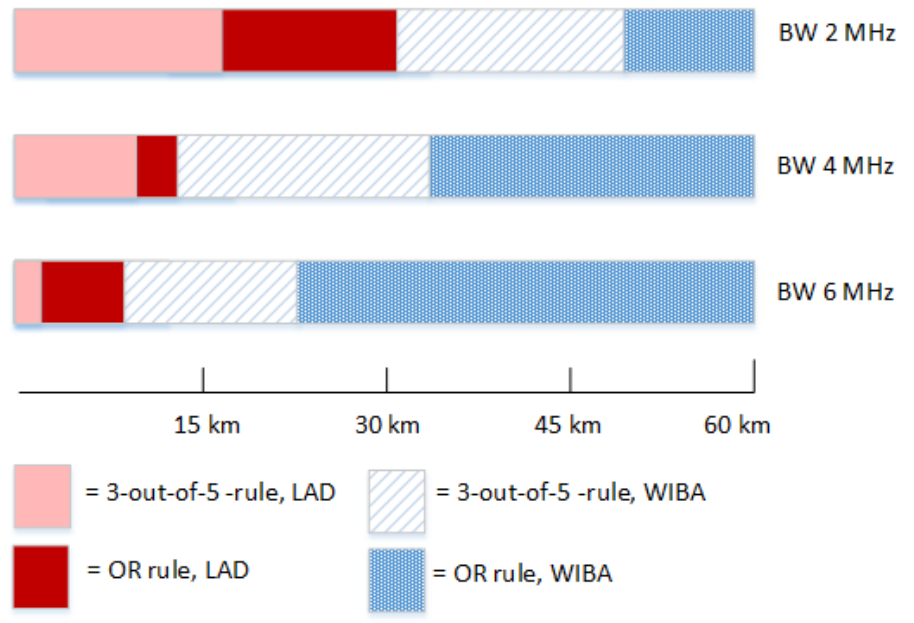

Figure 3. Distance $[\mathrm{km}]$ where cooperative sensing using OR and 3-out-of-5 -rules can detect the signal. Transmit power is $53 \mathrm{dBm}$ and signal $\mathrm{BW}$ is 2 , 4 and $6 \mathrm{MHz}$. The WIBA and LAD methods.

Transmit power $46 \mathrm{dBm}$

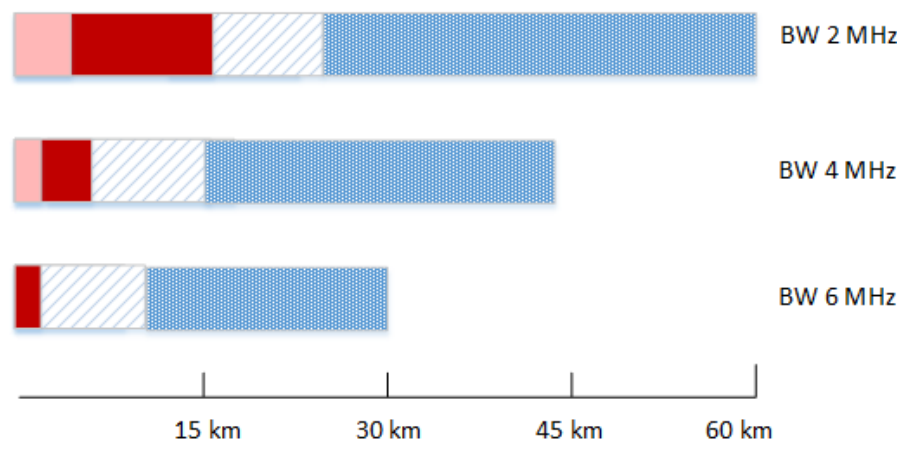

Figure 4. Distance $[\mathrm{km}]$ where cooperative sensing using OR and 3-out-of-5 -rules can detect the signal. Transmit power is $46 \mathrm{dBm}$ and signal BW is 2 , 4 and $6 \mathrm{MHz}$. The WIBA and LAD methods.

a half-fold and at its best five-fold when comparing to single sensing. When there are only few cooperative nodes $(3 / 5-$ rule), performance difference is $147-200 \%$ when compared to single sensing.

Figures 3 and 4 show visually the distance where cooperative sensing can detect 2,4 and $6 \mathrm{MHz}$ signals with transmit powers 53 and $46 \mathrm{dBm}$. Results for both OR and $3 / 5$-rules are presented. Although the detection area is a circle with radius $R$ $[\mathrm{km}]$, a rectangle shape is used here for the sake or simplicity. It can be seen that the WIBA method outperforms the LAD method clearly. As expected, cooperative OR sensing gives best results. It can also be seen that cooperative sensing gain increases with bandwidth of the signal to be detected. In the case of the WIBA method, cooperative sensing gives $26-45$ $\mathrm{km}$ (OR method) and $4-16 \mathrm{~km}(3 / 5-$-rule) benefit when compared to single sensing, depending on the signal $\mathrm{BW}$ and transmit power.

\section{CONCLUSION}

Cooperative spectrum sensing performance of the WIBA energy detection method was studied here to maximize signal detection distance in a rural area case using a dedicated channel model. Both OR and $k$-out-of- $n$ cooperative decision rules were studied to define required individual user detection probabilities to get final cooperative detection probability, $P_{d} \geq 0.9$. The signal detection distance results were compared to that of the LAD method. The WIBA method clearly outperformed the LAD method: the detection distance difference was tens of kilometers, even when the number of cooperative nodes was small. It was also noticed that cooperation improves the signal detection distance even five-fold, depending on the signal bandwidth, used cooperative decision rule, and the number of nodes. Therefore, the results are useful to enable proper design of cooperative spectrum sensing which helps to improve connectivity in remote areas together with the database approach.

\section{ACKNOWLEDGMENT}

This research has received funding from the European Union Horizon 2020 Programme (H2020/2017-2019) under grant agreement N0. 777137 and from the Ministry of Science, Technology under the 4th EU-BR Coordinated Call Information and Communication Technologies through 5GRANGE project. In addition, this research has been financially supported in part by Academy of Finland 6Genesis Flagship (grant 318927).

\section{REFERENCES}

[1] O. Meena and A. Somkuwar, "Comparative analysis of information fusion techniques for cooperative spectrum sensing in cognitive radio networks," Recent Trends in Information, Telecommunication and Computing, 2014.

[2] S. M. Mishra, A. Sahai, and R. W. Brodersen, "Cooperative sensing among cognitive radios," in IEEE International Conference on Communications, 2005, pp. 1-7.

[3] I. F. Akyildiz, B. F. Lo, and R. Balakrishnan, "Cooperative spectrum sensing in cognitive radio networks: A survey," Physical Communication, vol. 4, no. 1, 2011, pp. 40-62.

[4] H. Urkowitz, "Energy detection of unknown deterministic signals," Proceedings of the IEEE, vol. 55, 1967, pp. 523-531.

[5] S. Bae, J. So, and H. Kim, "On optimal cooperative sensing with energy detection in cognitive radio," Sensors (Basel), vol. 17, no. 9, 2017.

[6] H. Saarnisaari and J. Vartiainen, "Spectrum window based signal detection at low SNR," in International Conference on Military Communications and Information Systems (ICMCIS), Poland, Nov. 2018.

[7] J. Vartiainen, J. J. Lehtomäki, and H. Saarnisaari, "Double-threshold based narrowband signal extraction," in Vehicular Technology Conference (VTC), Stockholm, Sweden, May/June 2005, pp. 1288-1292.

[8] J. Vartiainen, "Concentrated signal extraction using consecutive mean excision algorithms," Ph.D. dissertation, Acta Univ Oul Technica C 368. Faculty of Technology, University of Oulu, Finland, Nov. 2010, http://jultika.oulu.fi/Record/isbn978-951-42-6349-1 [retrieved: Jun, 2019].

[9] Ericsson and Telstra, "Measurements of Extreme Rural Scenarios," report R1-166599, October 2016, release 15.

[10] B. Sokal and et al, "D3.1 Physical layer of the 5G-RANGE Part I," 5G-RANGE Project, Tech. Rep., 2018.

[11] Ericsson and Telstra, "Channel model for extreme rural scenario," report R1-167451, October 2016, release 15.

[12] 3GPP, "Study on channel model for frequencies from 0.5 to $100 \mathrm{GHz}$," 06 2018, release 14 . 
[13] Z. Khan, "Coordination and adaptation techniques for efficient resource utilization in cognitive radio networks," Ph.D. dissertation, Acta Univ Oul Technica C 395. Faculty of Technology, University of Oulu, Finland, Nov. 2011, http://urn.fi/urn:isbn:9789514295980 [retrieved: Jun, 2019],.

[14] T. Yucek and H. Arslan, "A survey of spectrum sensing algorithms for cognitive radio applications," IEEE Communications Surveys and Tutorials, vol. 11, no. 1, 2009.

[15] Y. Zou, Y.-D. Yao, and B. Zheng, "Cooperative relay techniques for cognitive radio systems: Spectrum sensing and secondary user transmissions," IEEE Communicatons Magazine, vol. 4, 2012.

[16] S. Atapattu, C. Tellambura, and H. Jiang, "Energy detection based cooperative spectrum sensing in cognitive radio networks," IEEE Transactions on Wireless Communications, vol. 10, no. 4, 2011, pp. 12321241.

[17] S. Chaudhari, J. Lunden, V. Koivunen, and H. V. Poor, "Cooperative sensing with imperfect reporting channels: Hard decisions or soft decisions?" IEEE Transactions on Signal Processing, vol. 60, no. 1, 2012, pp. 18-28.

[18] S. Althunibat and F. Granelli, "An objection-based collaborative spectrum sensing for cognitive radio networks," IEEE Communications Letter, vol. 18, 2014.

[19] Y. Wu, Y. Liu, W. RFan, F. Li, and S. Ji, "Is the OR rule a good choice for cooperative sensing in cognitive radio networks?" in 7th International Conference on Wireless Communications, Networking and Mobile Computing (WiCOM), 2011, pp. 1-4.

[20] J. Proakis, Digital Communications. New York, USA: McGraw-Hill, 1995.

[21] H. Saarnisaari and P. Henttu, "Impulse detection and rejection methods for radio systems," in Military Communications Conference (MILCOM), Boston, MA, USA, Oct. 2003.

[22] H. Saarnisaari, P. Henttu, and M. Juntti, "Iterative multidimensional impulse detectors for communications based on the classical diagnostic methods," IEEE Transactions on Communications, vol. 53, no. 3, Mar. 2005, pp. 395-398. 